\title{
Wine and other alcohol consumption and risk of ovarian cancer in the California Teachers Study cohort
}

\author{
Ellen T. Chang $\cdot$ Alison J. Canchola $\cdot$ Valerie S. Lee $\cdot$ Christina A. Clarke \\ David M. Purdie · Peggy Reynolds · Leslie Bernstein · Daniel O. Stram • \\ Hoda Anton-Culver · Dennis Deapen · Harvey Mohrenweiser • \\ David Peel · Rich Pinder · Ronald K. Ross · Dee W. West · William Wright \\ Argyrios Ziogas $\cdot$ Pamela L. Horn-Ross
}

Received: 24 May 2006/ Revised: 29 September 2006

(C) Springer Science+Business Media B.V. 2006

\begin{abstract}
Objective Whether alcohol consumption influences ovarian cancer risk is unclear. Therefore, we investigated the association between alcohol intake at various ages and risk of ovarian cancer.

Methods Among 90,371 eligible members of the California Teachers Study cohort who completed a baseline alcohol assessment in 1995-1996, 253 women were diagnosed with epithelial ovarian cancer by the end of 2003. Multivariate Cox proportional hazards
\end{abstract}

Ronald K. Ross Deceased

E. T. Chang $(\bowtie)$. A. J. Canchola · V. S. Lee .

C. A. Clarke · D. M. Purdie · P. Reynolds .

D. W. West · P. L. Horn-Ross

Northern California Cancer Center, 2201 Walnut Avenue,

Suite 300, Fremont, CA 94538, USA

e-mail: echang@nccc.org

E. T. Chang · C. A. Clarke · P. Reynolds .

D. W. West · P. L. Horn-Ross

Department of Health Research and Policy, Stanford

University School of Medicine, Stanford, CA, USA

L. Bernstein · D. O. Stram · D. Deapen .

R. Pinder $\cdot$ R. K. Ross

Norris Comprehensive Cancer Center, Keck School of Medicine of the University of Southern California, Los Angeles, CA, USA

H. Anton-Culver · H. Mohrenweiser · D. Peel .

A. Ziogas

School of Medicine, University of California, Irvine,

CA, USA

W. Wright

Cancer Surveillance Section, California Department of

Health Services, Sacramento, CA, USA regression analysis was performed to estimate relative risks (RRs) and 95\% confidence intervals (CIs).

Results Consumption of total alcohol, beer, or liquor in the year prior to baseline, at ages 30-35 years, or at ages 18-22 years was not associated with risk of ovarian cancer. Consumption of at least one glass per day of wine, compared to no wine, in the year before baseline was associated with increased risk of developing ovarian cancer: $\mathrm{RR}=1.57$ (95\% CI 1.11-2.22), $P_{\text {trend }}=0.01$. The association with wine intake at baseline was particularly strong among peri-/postmenopausal women who used estrogen-only hormone therapy and women of high socioeconomic status.

Conclusions Alcohol intake does not appear to affect ovarian cancer risk. Constituents of wine other than alcohol or, more likely, unmeasured determinants of wine drinking were associated with increased risk of ovarian cancer.

Keywords Ovarian cancer - Alcoholic beverages . Cohort studies · Women's health

\section{Introduction}

Consistent evidence that moderate alcohol consumption increases the risk of breast cancer, presumably by elevating estrogen and androgen levels [1], suggests that alcohol intake might also influence the development of other hormone-related malignancies, such as ovarian cancer. From a public health standpoint, this hypothesis is attractive since alcohol consumption could represent a readily modifiable ovarian cancer risk factor, whereas other risk factors, particularly reproductive characteristics [2, 3], are generally less 
amenable to change. Findings from previous studies examining the association between alcohol and ovarian cancer risk have been discrepant, with published reports of mostly null [3-19], some positive [15, 16, 20, 21], and some inverse associations [14, 17, 22-25], including occasional variation in associations by type of alcohol and/or subgroup of ovarian cancer cases. No systematic differences in findings are apparent between case-control and cohort studies.

Although ethanol itself is not a direct ovarian carcinogen, it could influence ovarian cancer risk through effects on steroid hormones, especially estrogens, which are believed to play a primary role in ovarian carcinogenesis [26]. In pre-menopausal women, moderate to high alcohol consumption is associated with elevated levels of total and bioavailable estrogens and androgens [27-29], reduced fertility [30, 31], and decreased menstrual cycle variability and length, resulting in increased cumulative estrogen exposure [32]. In post-menopausal women, moderate alcohol intake markedly increases circulating estrogen levels in hormone therapy (HT) users [33, 34], and it may elevate estrogen and androgen levels in women not using HT [35]. Other mechanisms for alcohol-related carcinogenesis include alteration of gonadotropin levels, promotion of DNA damage, impaired folate metabolism and DNA hypomethylation, inhibition of carcinogen detoxification or clearance, and increased metastatic potential of tumor cells [1].

Given the biologic plausibility of a role of alcohol intake in ovarian cancer etiology, we examined the association between alcohol consumption and risk of ovarian cancer in a prospective cohort in which baseline alcohol consumption was associated with increased breast cancer risk [36, 37]. With data on past and baseline intake of specific alcoholic beverages, as well as information on a variety of demographic and behavioral factors, we were also able to explore the importance of drinking patterns and potential effect modifiers of any association.

\section{Methods}

\section{Study population}

The California Teachers Study (CTS) cohort includes 133,479 active and retired female public school teachers and administrators who were members of the California State Teachers Retirement System and returned a mailed questionnaire in 1995-1996 [38]. The questionnaire assessed a range of potential cancer risk factors including menstrual and reproductive history, personal and family medical history, physical activity, dietary intake during the previous year (using a foodfrequency questionnaire and portion-size assessment [39-42]), alcohol and tobacco use, and other factors. All women provided written informed consent to participate in the study, and the study protocol was approved by the institutional review boards of all participating institutions.

For this analysis, we excluded women (in a hierarchical manner) as follows: those who 1.) lived outside of California at baseline $(n=8,867)$; 2 .) did not provide adequate information on personal history of cancer $(n=662) ; 3$. $)$ consented to participate only in analyses of breast cancer $(n=18)$; 4.) reported having had ovarian cancer before baseline or were identified by the California Cancer Registry as having been previously diagnosed with ovarian cancer $(n=640)$; 5.) reported having had a bilateral oophorectomy before baseline $(n=14,422) ; 6$.) were aged 85 years or older at baseline $(n=1,874)$; 7.) reported never having had a first menstrual period $(n=51)$; 8.) provided multiple invalid, inconsistent, or blank responses to the dietary questionnaire $(n=2,942)$; 9.) reported food consumption that was judged to be implausibly low (i.e., $<600$ calories/day) or high (i.e., $>5,000$ calories/day) ( $n=1,565$ and 69 , respectively); or 10. ) provided invalid, missing, or inconsistent data with respect to alcohol intake during the previous year $(n=5,094)$ or the earlier two periods evaluated $(n=6,904)$. Of the 90,371 remaining women included in this analysis, 227 were diagnosed with invasive epithelial ovarian cancer and 26 were diagnosed with borderline epithelial ovarian cancer (ICD-O-3 site C569, excluding non-epithelial ovarian cancer cases [morphology codes 8240-8245, 8590-8671, and 90609989] [43]) after joining the cohort and on or before 31 December 2003.

\section{Alcohol assessment}

Participants reported average weekly consumption of beer, wine/champagne, and cocktails/liquor at ages 1822 years, at ages $30-35$ years, and in the year prior to baseline. Available response categories for average number of drinks per week were none, $\leq 3,4-10$, $11-17,18-24$, and $\geq 25$. A typical drink was defined as one bottle, can, or glass of beer; one glass of wine, champagne, or wine cooler; or one cocktail, shot, or mixed drink of liquor. A single drink of beer, wine, or liquor was assumed to contain 13.2,11.1, or 15.0 grams of alcohol, respectively. Based on these standards, daily intake of grams of alcohol from each type of drink was calculated for each woman during each time 
period. Alcohol intake in the cohort was reproducible $(\mathrm{r}=0.87)$ and valid compared to multiple 24-hour recalls $(r=0.74)$ (Pamela Horn-Ross, unpublished data).

Daily consumption of alcohol from beer was categorized as $0,<13.2$, or $\geq 13.2$ grams/day; alcohol from wine was categorized as $0,<11.1$, or $\geq 11.1$ grams/day; alcohol from liquor was categorized as $0,<15.0$, or $\geq 15.0$ grams/day; and total alcohol was categorized as $0,<10.0,10.0$ to $<20.0$, or $\geq 20.0$ grams/day. These categories were defined based on the grams of alcohol per standard drink, and on findings from previous analyses of alcohol intake and breast cancer risk in the CTS [36, 37].

Based on their drinking patterns in any two time periods (ages 18-22 years and baseline, ages 30-35 years and baseline, or ages 18-22 years and 30-35 years), women were categorized as having been non-drinkers, moderate drinkers (in the middle category or categories of intake), or heavy drinkers (in the highest category of intake) in both time periods; or decreasing or increasing drinkers if their intake changed between the earlier and the later time period. For each type of alcoholic drink and each time period, women also reported how many days per week they usually had at least one drink. Women were categorized as non-drinkers, as drinking alcohol on 1-4 days/ week, or as drinking alcohol on $\geq 5$ days/week [37].

\section{Residence-based measures}

Based on residential address at entry, women were geocoded to census block groups. To obtain a measure of each cohort member's relative socioeconomic status (SES), all 1990 census block groups in the state of California were ranked by three measures, according to deciles based on the statewide adult population: percentage of adults over age 25 years who had completed a college degree or higher; median family income; and percentage of adults employed in managerial/professional occupations [44]. A summary SES metric was created by adding the scores (1 through 10) across these attributes; participants were then categorized into deciles or quartiles of the total score. Women were also categorized by median family income in their census block group, based on deciles in the cohort.

Residential census block groups were categorized as rural, town, small city, metropolitan suburban, or metropolitan urban based on population size and density [44]. Women were also classified as residents of the Greater San Francisco Bay Area (Alameda, Contra Costa, Marin, Monterey, San Benito, San Francisco, San Mateo, Santa Clara, and Santa Cruz Counties); the
Southern Coastal area (Orange, Los Angeles, and San Diego Counties); or the rest of California [45].

Follow-up

Person-time was accrued from the date of completion of the baseline questionnaire until the date of first diagnosis with borderline or invasive ovarian cancer, relocation out of California, death, or 31 December 2003, whichever occurred earliest. Information on incident ovarian cancer and tumor characteristics was obtained through annual linkage of cohort members to the California Cancer Registry (CCR) based on full name, date of birth, address, and social security number, including manual review of possible matches. The CCR is the population-based cancer registry that covers the entire state of California, has agreements with 13 other states for case-sharing purposes, and maintains high-quality data standards as part of the National Cancer Institute's Surveillance, Epidemiology, and End Results program. Reporting of new cancer diagnoses to the CCR has been mandated by California state law since 1985, and coverage is estimated to be $99 \%$ complete [46], such that all members of the CTS cohort are effectively in active follow-up for cancer outcomes as long as they reside in California.

Date and cause of death are ascertained through linkages with the California state mortality file and the national Social Security Administration death master file, as well as reports from relatives. Address changes are obtained through annual mailed newsletters, notifications by participants, and record linkages with the California Department of Motor Vehicles, the US Postal Service National Change of Address database, and other sources.

\section{Statistical analysis}

Multivariable Cox proportional hazards regression analysis was performed to evaluate the association between alcohol consumption and risk of ovarian cancer, using ages at the start and end of follow-up (in days) to define the time scale. Models were adjusted for race (White or non-White), total daily caloric intake (continuous), parity ( $0,1-2$, or $\geq 3$ full-term pregnancies), use of oral contraceptives (never, $<5$ years, or $\geq 5$ years), average strenuous physical exercise $(<0.5,0.5-$ 3.99 , or $\geq 4$ hours/week during lifetime up to age 54 years), menopausal status/use of HT (pre-menopausal, unknown menopausal status, or peri-/postmenopausal and: never used HT, used combination estrogen-progestin HT, used a mixture of combination 
and estrogen-only ("mixed") HT, used estrogen-only HT for $\leq 5$ years, used estrogen-only HT for $>5$ years, used estrogen-only HT for an unknown duration, or unknown HT use), and an interaction between menopausal status/HT use and the time scale (because menopausal status/HT use violated the proportionality assumption), and were stratified by age at baseline (in years). These potential confounders were chosen based on statistically significant ( $p$-value $\leq 0.05$ ) associations with risk of ovarian cancer, and on prior knowledge of ovarian cancer risk factors. Missing values were coded as dummy variables, none of which were found to be associated with ovarian cancer risk. We tested the assumption of proportional hazards for each alcohol variable using significance tests of interactions with the time scale and visual examination of scaled Schoenfeld residual plots [47], and found no violations of the proportionality assumption.

Hazard rate ratios, presented as relative risks (RR), and corresponding $95 \%$ confidence intervals $(\mathrm{CI})$ were estimated for each type of alcoholic beverage, comparing categories of consumption to non-drinkers of that alcohol type as the reference group. Tests for linear trend across exposure categories were conducted using the median of each category coded as an ordinal variable. The median of the highest category of beer, wine, or liquor intake was equal to the lower boundary because most women in those categories reported consuming one drink per day.

Analyses were first performed for all eligible women, and then repeated with restriction to women who were peri- or post-menopausal at baseline. In addition, analyses were performed with restriction to cases of invasive ovarian cancer (excluding borderline cases) or serous ovarian cancer (ICD-O-3 morphology codes 8441-8462 and 9014 [43]; $n=114)$. Likelihood ratio tests were used to evaluate homogeneity of the estimated RR between strata of women, as well as significant differences in fit between models with and without additional covariates. Tests for non-linearity of trend were based on a likelihood ratio test comparing models with the exposure coded as an ordinal or a categorical variable [48]. Associations with wine drinking were evaluated using multivariate logistic regression, using covariates as defined above. All analyses were performed using SAS Version 9.1 (SAS Institute, Cary, NC).

\section{Results}

Distributions of demographic characteristics and ovarian cancer risk factors in the eligible study cohort are shown in Table 1. The median length of follow-up was 2,959 days (8.1 years) and the median age of participants at baseline was 50 years. As shown in Table 2, there was no overall difference in risk of ovarian cancer by total alcohol consumption in the year before baseline, at ages 30-35 years, or at ages 18-22 years. Similarly, consumption of alcohol from beer or liquor during any of the three time periods was not significantly associated with risk of ovarian cancer. In contrast, intake of alcohol from wine during the year before baseline was associated with statistically significantly elevated risk of ovarian cancer. After adjusting for alcohol intake from beer and liquor, as well as ovarian cancer risk factors, women who drank at least 11.1 grams per day of alcohol from wine-the equivalent of one glass per day-at baseline were at $57 \%$ higher risk of ovarian cancer, compared to women who did not drink wine $\left(P_{\text {trend }}=0.01\right)$. Intake of alcohol from wine at ages 30-35 years or ages 18-22 years was not significantly associated with ovarian cancer risk, although the estimated RRs were consistent with those for baseline wine intake.

Intake of specific alcohol types, beyond total alcohol, was associated with ovarian cancer risk, as assessed by comparing a multivariate model with beer, wine, and alcohol consumption in the year before baseline to a model with alcohol consumption alone $(p=0.05,2$ d.f.). Furthermore, the association of wine consumption with ovarian cancer risk differed from that of beer or liquor consumption, as assessed by comparing a multivariate model with beer/liquor and wine consumption to a model with alcohol consumption ( $p=0.02,1$ d.f.). Controlling for total alcohol intake did not attenuate the positive association between wine intake at any time period and risk of ovarian cancer (data not shown). Women who drank wine only in the year before baseline (median daily alcohol intake $=11.1$ grams), relative to non-drinkers, had an ovarian cancer RR of 1.40 (95\% CI 1.01-1.93). In contrast, risk did not vary between non-drinkers and women who drank beer or liquor only (median daily intake of alcohol $=4.5$ grams; $\mathrm{RR}=1.03[95 \% \mathrm{CI}$ $0.58-1.83]$ ) or beer/liquor and wine (median daily alcohol intake $=11.8$ grams; $\mathrm{RR}=1.08$ [95\% CI 0.79 1.48]). The multivariate $\mathrm{RR}$ among women who exclusively drank at least one glass of wine per day, compared to non-drinkers, was 1.70 (95\% CI 1.10 2.62). After simultaneously adjusting for wine drinking during the year before baseline and at ages 18-22 years and 30-35 years, the RR associated with drinking at least one daily glass of wine at baseline was $1.33(95 \%$ CI 0.91-1.96); at ages 30-35 years, RR $=1.01(95 \%$ CI $0.61-1.68)$; and at ages $18-22$ years, $\mathrm{RR}=1.28(95 \%$ CI 0.58-2.87). 
Table 1 Selected baseline characteristics of the California Teachers Study (CTS) cohort included in the present analysis $(n=90,371)$

\begin{tabular}{|c|c|c|}
\hline Characteristic & $n$ & $(\%)$ \\
\hline \multicolumn{3}{|l|}{ Age at baseline (years) } \\
\hline$<35$ & 10,456 & $(11.6 \%)$ \\
\hline $35-44$ & 18,546 & $(20.5 \%)$ \\
\hline $45-54$ & 28,275 & $(31.3 \%)$ \\
\hline $55-64$ & 16,229 & $(18.0 \%)$ \\
\hline $65-74$ & 11,496 & $(12.7 \%)$ \\
\hline $75-84$ & 5,369 & $(5.9 \%)$ \\
\hline \multicolumn{3}{|l|}{ Race/ethnicity } \\
\hline White & 78,468 & $(86.8 \%)$ \\
\hline Non-white & 11,266 & $(12.5 \%)$ \\
\hline Unknown & 637 & $(0.7 \%)$ \\
\hline \multicolumn{3}{|l|}{ Parity (full-term pregnancies) } \\
\hline None & 23,810 & $(26.3 \%)$ \\
\hline $1-2$ & 43,674 & $(48.3 \%)$ \\
\hline$\geq 3$ & 21,412 & $(23.7 \%)$ \\
\hline Unknown & 1,475 & $(1.6 \%)$ \\
\hline \multicolumn{3}{|l|}{ Oral contraceptive use (years) } \\
\hline None & 26,643 & $(29.5 \%)$ \\
\hline$<5$ & 27,608 & $(30.5 \%)$ \\
\hline$\geq 5$ & 32,463 & $(35.9 \%)$ \\
\hline Unknown & 3,657 & $(4.0 \%)$ \\
\hline \multicolumn{3}{|c|}{ Lifetime strenuous physical activity (average hours/week) } \\
\hline$<0.5$ & 25,254 & $(27.9 \%)$ \\
\hline $0.5-3.9$ & 49,467 & $(54.7 \%)$ \\
\hline$\geq 4.0$ & 15,293 & $(16.9 \%)$ \\
\hline Unknown & 357 & $(0.4 \%)$ \\
\hline \multicolumn{3}{|l|}{ Menopausal status } \\
\hline Pre-menopausal & 42,204 & $(46.7 \%)$ \\
\hline Peri-menopausal & 2,202 & $(2.4 \%)$ \\
\hline Post-menopausal & 39,744 & $(44.0 \%)$ \\
\hline Unknown & 6,221 & $(6.9 \%)$ \\
\hline \multicolumn{3}{|c|}{ Hormone therapy (HT) use (peri-/post-menopausal women only) } \\
\hline None & 12,968 & $(30.9 \%)$ \\
\hline Combination estrogen + progestin $\mathrm{HT}$ & 14,235 & $(33.9 \%)$ \\
\hline Estrogen-only $\mathrm{HT}, \leq 5$ years & 4,342 & $(10.4 \%)$ \\
\hline Estrogen-only $\mathrm{HT},>5$ years & 4,899 & $(11.7 \%)$ \\
\hline Estrogen-only HT, unknown duration & 332 & $(0.8 \%)$ \\
\hline Mixed combination and estrogen-only HT & 4,776 & $(11.4 \%)$ \\
\hline Unknown & 394 & $(0.9 \%)$ \\
\hline \multicolumn{3}{|l|}{ Region of residence within California ${ }^{a}$} \\
\hline Greater San Francisco Bay Area & 18,444 & $(20.4 \%)$ \\
\hline Southern Coastal/Los Angeles Area & 35,388 & $(39.2 \%)$ \\
\hline Other areas & 36,483 & $(40.4 \%)$ \\
\hline Unknown & 56 & $(0.1 \%)$ \\
\hline \multicolumn{3}{|l|}{ Type of residence } \\
\hline Rural & 12,677 & $(14.0 \%)$ \\
\hline Town & 3,136 & $(3.5 \%)$ \\
\hline Small city & 16,135 & $(17.9 \%)$ \\
\hline Metropolitan suburban & 48,278 & $(53.4 \%)$ \\
\hline Metropolitan urban & 9,038 & $(10.0 \%)$ \\
\hline Unknown & 1,107 & $(1.2 \%)$ \\
\hline \multicolumn{3}{|c|}{ Statewide percentile of socioeconomic status in census block group ${ }^{a}$} \\
\hline$\leq 49$ th & 18,962 & $(21.0 \%)$ \\
\hline $50-59$ th & 10,199 & $(11.3 \%)$ \\
\hline $60-69$ th & 12,620 & $(14.0 \%)$ \\
\hline $70-79$ th & 14,508 & $(16.1 \%)$ \\
\hline $80-89$ th & 16,864 & $(18.7 \%)$ \\
\hline $90-99$ th & 16,076 & $(17.8 \%)$ \\
\hline Unknown & 1,142 & $(1.3 \%)$ \\
\hline
\end{tabular}


Table 1 continued

\begin{tabular}{lrr}
\hline Characteristic & $n$ & \\
\hline Smoking history & & \\
Never & 60,868 & $(67.4 \%)$ \\
Former & 24,989 & $(27.7 \%)$ \\
Current & 4,428 & $(4.9 \%)$ \\
Unknown & 86 & $(0.1 \%)$ \\
Alcohol drinking in the year prior to baseline & 31,024 & $(34.3 \%)$ \\
None & 2,222 & $(2.5 \%)$ \\
Beer only & 19,365 & $(21.4 \%)$ \\
Wine only & 2,868 & $(3.2 \%)$ \\
Liquor only & 1,005 & $(1.1 \%)$ \\
Beer and liquor only & 33,887 & $(37.5 \%)$ \\
Wine and beer/liquor & & \\
\hline
\end{tabular}

${ }^{a}$ See Methods for definition

Table 2 Relative risks (RRs) and 95\% confidence intervals (CIs) for associations between intake of specific types of alcohol at various ages and risk of ovarian cancer

\begin{tabular}{|c|c|c|c|c|c|c|c|c|}
\hline $\begin{array}{l}\text { Alcohol } \\
\text { type }\end{array}$ & $\begin{array}{l}\text { Time } \\
\text { period }\end{array}$ & $\begin{array}{l}\text { Daily intake } \\
\text { (g/day) }\end{array}$ & $\begin{array}{l}\text { Median } \\
\text { (g/day) }\end{array}$ & Cases $(n)$ & $\mathrm{RR}^{\mathrm{a}}$ & $95 \% \mathrm{CI}^{\mathrm{a}}$ & $\mathrm{RR}^{\mathrm{b}}$ & $95 \% \mathrm{CI}^{\mathrm{b}}$ \\
\hline \multicolumn{9}{|c|}{ Total alcohol } \\
\hline & \multicolumn{8}{|c|}{ Year before baseline } \\
\hline & & None & 0.0 & 77 & 1.00 & (reference) & - & \\
\hline & & $<10.0$ & 4.5 & 81 & 1.04 & $(0.76,1.42)$ & - & \\
\hline & & $10.0-<20.0$ & 11.8 & 72 & 1.47 & $(1.06,2.03)$ & - & \\
\hline & & $\geq 20.0$ & 28.2 & 23 & 1.15 & $(0.71,1.84)$ & - & \\
\hline & & & & & \multicolumn{4}{|c|}{$P_{\text {trend }}=0.19$} \\
\hline & \multicolumn{8}{|c|}{ Ages 30-35 years ${ }^{\mathrm{c}}$} \\
\hline & & None & 0.0 & 67 & 1.00 & (reference) & - & \\
\hline & & $<10.0$ & 7.3 & 101 & 1.14 & $(0.83,1.56)$ & - & \\
\hline & & $10.0-<20.0$ & 11.8 & 47 & 1.08 & $(0.74,1.59)$ & - & \\
\hline & & $\geq 20.0$ & 29.7 & 16 & 0.99 & $(0.56,1.71)$ & - & \\
\hline & & & & & \multicolumn{4}{|c|}{$P_{\text {trend }}=0.99$} \\
\hline & \multicolumn{8}{|c|}{ Ages $18-22$ years $^{\mathrm{d}}$} \\
\hline & & None & 0.0 & 131 & 1.00 & (reference) & - & \\
\hline & & $<10.0$ & 4.5 & 62 & 0.76 & $(0.55,1.03)$ & - & \\
\hline & & $10.0-<20.0$ & 11.8 & 36 & 1.26 & $(0.86,1.84)$ & - & \\
\hline & & $\geq 20.0$ & 28.8 & 9 & 1.00 & $(0.50,1.99)$ & - & \\
\hline & & & & & \multicolumn{4}{|c|}{$P_{\text {trend }}=0.63$} \\
\hline \multicolumn{9}{|l|}{ Beer } \\
\hline & \multicolumn{8}{|c|}{ Year before baseline } \\
\hline & & None & 0.0 & 199 & 1.00 & (reference) & 1.00 & (reference) \\
\hline & & $<13.2$ & 4.0 & 51 & 0.96 & $(0.70,1.31)$ & 0.89 & $(0.64,1.24)$ \\
\hline & & $\geq 13.2$ & 13.2 & 3 & 0.58 & $(0.19,1.84)$ & 0.54 & $(0.17,1.70)$ \\
\hline & & & & & \multicolumn{2}{|c|}{$P_{\text {trend }}=0.40$} & \multicolumn{2}{|c|}{$P_{\text {trend }}=0.22$} \\
\hline & \multicolumn{8}{|c|}{ Ages $30-35$ years $^{\mathrm{c}}$} \\
\hline & & None & 0.0 & 180 & 1.00 & (reference) & 1.00 & (reference) \\
\hline & & $<13.2$ & 4.0 & 45 & 0.81 & $(0.58,1.13)$ & 0.75 & $(0.53,1.06)$ \\
\hline & & $\geq 13.2$ & 13.2 & 6 & 0.72 & $(0.32,1.64)$ & 0.73 & $(0.32,1.69)$ \\
\hline & & & & & \multicolumn{2}{|c|}{$P_{\text {trend }}=0.20$} & \multicolumn{2}{|c|}{$P_{\text {trend }}=0.16$} \\
\hline & \multicolumn{8}{|c|}{ Ages $18-22$ years $^{\mathrm{d}}$} \\
\hline & & None & 0.0 & 179 & 1.00 & (reference) & 1.00 & (reference) \\
\hline & & $<13.2$ & 4.0 & 46 & 0.95 & $(0.68,1.32)$ & 0.93 & $(0.65,1.33)$ \\
\hline & & $\geq 13.2$ & 13.2 & 13 & 1.21 & $(0.68,2.16)$ & 1.30 & $(0.70,2.39)$ \\
\hline & & & & & \multicolumn{2}{|c|}{$P_{\text {trend }}=0.67$} & \multicolumn{2}{|c|}{$P_{\text {trend }}=0.57$} \\
\hline
\end{tabular}


Table 2 continued

\begin{tabular}{|c|c|c|c|c|c|c|c|c|}
\hline $\begin{array}{l}\text { Alcohol } \\
\text { type }\end{array}$ & $\begin{array}{l}\text { Time } \\
\text { period }\end{array}$ & $\begin{array}{l}\text { Daily intake } \\
\text { (g/day) }\end{array}$ & $\begin{array}{l}\text { Median } \\
\text { (g/day) }\end{array}$ & Cases $(n)$ & $\mathrm{RR}^{\mathrm{a}}$ & $95 \% \mathrm{CI}^{\mathrm{a}}$ & $\mathrm{RR}^{\mathrm{b}}$ & $95 \% \mathrm{CI}^{\mathrm{b}}$ \\
\hline \multicolumn{9}{|l|}{ Wine } \\
\hline & \multicolumn{8}{|c|}{ Year before baseline } \\
\hline & & None & 0.0 & 91 & 1.00 & (reference) & 1.00 & (reference) \\
\hline & & $<11.1$ & 3.3 & 99 & 1.08 & $(0.81,1.43)$ & 1.09 & $(0.80,1.50)$ \\
\hline & & $\geq 11.1$ & 11.1 & 63 & 1.50 & $(1.08,2.09)$ & 1.57 & $(1.11,2.22)$ \\
\hline & & & & & \multicolumn{2}{|c|}{$P_{\text {trend }}=0.01^{\mathrm{e}}$} & \multicolumn{2}{|c|}{$P_{\text {trend }}=0.01^{\mathrm{e}}$} \\
\hline & \multicolumn{4}{|c|}{ Ages $30-35$ years $^{c}$} & & & & \\
\hline & & None & 0.0 & 90 & 1.00 & (reference) & 1.00 & (reference) \\
\hline & & $<11.1$ & 3.3 & 112 & 1.19 & $(0.90,1.59)$ & 1.26 & $(0.92,1.71)$ \\
\hline & & $\geq 11.1$ & 11.1 & 29 & 1.21 & $(0.78,1.86)$ & 1.38 & $(0.87,2.19)$ \\
\hline & \multirow{2}{*}{\multicolumn{4}{|c|}{ Ages $18-22$ years $^{\mathrm{d}}$}} & \multicolumn{2}{|c|}{$P_{\text {trend }}=0.36$} & \multicolumn{2}{|c|}{$P_{\text {trend }}=0.16$} \\
\hline & & & & & & & & \\
\hline & & None & 0.0 & 167 & 1.00 & (reference) & 1.00 & (reference) \\
\hline & & $<11.1$ & 3.3 & 63 & 1.11 & $(0.82,1.50)$ & 1.17 & $(0.84,1.63)$ \\
\hline & & $\geq 11.1$ & 11.1 & 8 & 1.42 & $(0.69,2.91)$ & 1.63 & $(0.76,3.50)$ \\
\hline & & & & & \multicolumn{2}{|c|}{$P_{\text {trend }}=0.28$} & \multicolumn{2}{|c|}{$P_{\text {trend }}=0.15$} \\
\hline \multirow[t]{16}{*}{ Liquor } & \multirow{2}{*}{\multicolumn{8}{|c|}{ Year before baseline }} \\
\hline & & & & & & & & \\
\hline & & None & 0.0 & 169 & 1.00 & (reference) & 1.00 & (reference) \\
\hline & & $<15.0$ & 4.5 & 68 & 1.09 & $(0.82,1.45)$ & 1.06 & $(0.78,1.44)$ \\
\hline & & $\geq 15.0$ & 15.0 & 16 & 0.87 & $(0.52,1.47)$ & 0.82 & $(0.48,1.39)$ \\
\hline & & & & & \multicolumn{2}{|c|}{$P_{\text {trend }}=0.80$} & \multicolumn{2}{|c|}{$P_{\text {trend }}=0.56$} \\
\hline & \multicolumn{4}{|c|}{ Ages $30-35$ years $^{c}$} & & & & \\
\hline & & None & 0.0 & 124 & 1.00 & (reference) & 1.00 & (reference) \\
\hline & & $<15.0$ & 4.5 & 93 & 1.07 & $(0.81,1.41)$ & 1.04 & $(0.77,1.40)$ \\
\hline & & $\geq 15.0$ & 15.0 & 14 & 0.77 & $(0.44,1.35)$ & 0.75 & $(0.42,1.36)$ \\
\hline & & & & & \multicolumn{2}{|c|}{$P_{\text {trend }}=0.52$} & \multicolumn{2}{|c|}{$P_{\text {trend }}=0.44$} \\
\hline & \multicolumn{4}{|c|}{ Ages $18-22$ years $^{\mathrm{d}}$} & & & & \\
\hline & & None & 0.0 & 164 & 1.00 & (reference) & 1.00 & (reference) \\
\hline & & $<15.0$ & 4.5 & 69 & 0.99 & $(0.74,1.32)$ & 0.94 & $(0.68,1.29)$ \\
\hline & & $\geq 15.0$ & 15.0 & 5 & 0.62 & $(0.25,1.52)$ & 0.49 & $(0.19,1.26)$ \\
\hline & & & & & \multicolumn{2}{|c|}{$P_{\text {trend }}=0.39$} & \multicolumn{2}{|c|}{$P_{\text {trend }}=0.17$} \\
\hline
\end{tabular}

${ }^{a}$ Adjusted for race, total energy intake, parity, oral contraceptive use, strenuous exercise, and menopausal status/hormone therapy use; stratified by age at baseline

b Additionally adjusted for consumption of other alcohol types in the same time period

c Among women over age 35 years at baseline with non-missing data on alcohol consumption at ages 30-35 years

d Among women over age 22 years at baseline with non-missing data on alcohol consumption at ages 18-22 years

e $p$ for non-linearity of trend $>0.05$

Women who drank at least one glass per day of wine both in an earlier time period (18-22 years or 3035 years) and at baseline were at significantly higher risk of ovarian cancer, compared to wine non-drinkers (Table 3). Results were similar when based on intake at ages 18-22 years and 30-35 years (data not shown). Women who were heavy wine drinkers in all three time periods ( $n=5$ cases) had over four times the risk of ovarian cancer relative to women who never drank wine in any time period $(\mathrm{RR}=4.60[95 \% \mathrm{CI}=1.76-12.01])$, adjusting for beer and liquor drinking patterns. Marginally elevated risk of ovarian cancer was also observed among women who increased or decreased their wine intake between ages 18-22 years and either baseline or ages 30-35 years (latter data not shown). No patterns of total alcohol, beer, or liquor intake were significantly associated with ovarian cancer risk. Furthermore, ovarian cancer risk did not vary according to number of drinking days per week at baseline, whether for total alcohol, beer, wine, or liquor, although the risk of ovarian cancer rose slightly with increasing frequency of wine consumption at baseline $\left(P_{\text {trend }}=0.11\right)$.

The lack of an association with total alcohol, beer, or liquor intake, as well as the persistence of the association between wine intake and risk of ovarian cancer after adjustment for alcohol consumption, suggested that determinants of wine drinking, or ingredients of wine other than alcohol, were responsible for the observed positive association. After mutual adjustment, older age (up to ages 65-69 years), White 
Table 3 Relative risks (RRs) and 95\% confidence intervals (CIs) for associations between patterns of drinking specific types of alcohol and risk of ovarian cancer

\begin{tabular}{|c|c|c|c|c|c|c|c|}
\hline Alcohol type & Time period & Drinking pattern & Cases $(n)$ & $\mathrm{RR}^{\mathrm{a}}$ & $95 \% \mathrm{CI}^{\mathrm{a}}$ & $\mathrm{RR}^{\mathrm{b}}$ & $95 \% \mathrm{CI}^{\mathrm{b}}$ \\
\hline \multicolumn{8}{|l|}{ Total alcohol } \\
\hline & \multicolumn{7}{|c|}{ Age $30-35$ years and the year before baseline ${ }^{c}$} \\
\hline & & Never & 49 & 1.00 & (reference) & - & \\
\hline & & Steady moderate & 118 & 1.25 & $(0.89,1.76)$ & - & \\
\hline & & Decreasing & 29 & 1.06 & $(0.67,1.68)$ & - & \\
\hline & & Increasing & 27 & 1.10 & $(0.68,1.77)$ & - & \\
\hline & & Steady heavy & 8 & 1.32 & $(0.62,2.82)$ & - & \\
\hline & \multicolumn{7}{|c|}{ Age $18-22$ years and the year before baseline ${ }^{\mathrm{d}}$} \\
\hline & & Never & 55 & 1.00 & (reference) & - & \\
\hline & & Steady moderate & 70 & 1.07 & $(0.74,1.53)$ & - & \\
\hline & & Decreasing & 24 & 1.06 & $(0.65,1.72)$ & - & \\
\hline & & Increasing & 86 & 1.30 & $(0.92,1.84)$ & - & \\
\hline & & Steady heavy & 3 & 1.87 & $(0.58,6.04)$ & - & \\
\hline & \multicolumn{7}{|c|}{ Year before baseline } \\
\hline & & 0 days/week & 77 & 1.00 & (reference) & - & \\
\hline & & 1 to 4 days/week & 98 & 1.25 & $(0.92,1.69)$ & - & \\
\hline & & 5 to 7 days/week, $\leq 20 \mathrm{~g} /$ day & 30 & 1.36 & $(0.88,2.08)$ & - & \\
\hline & & 5 to 7 days/week, $>20 \mathrm{~g} /$ day & 18 & 1.14 & $(0.68,1.93)$ & - & \\
\hline \multicolumn{8}{|c|}{ 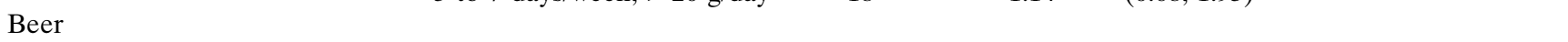 } \\
\hline & \multicolumn{7}{|c|}{ Age $30-35$ years and the year before baseline ${ }^{c}$} \\
\hline & & Never & 163 & 1.00 & (reference) & 1.00 & (reference) \\
\hline & & Steady moderate & 26 & 0.80 & $(0.53,1.21)$ & 0.73 & $(0.47,1.13)$ \\
\hline & & Decreasing & 25 & 0.94 & $(0.62,1.44)$ & 0.88 & $(0.57,1.38)$ \\
\hline & & Increasing & 17 & 1.08 & $(0.65,1.79)$ & 0.99 & $(0.59,1.66)$ \\
\hline & & Steady heavy & 0 & - & & - & \\
\hline & \multicolumn{7}{|c|}{ Age $18-22$ years and the year before baseline ${ }^{\mathrm{d}}$} \\
\hline & & Never & 159 & 1.00 & (reference) & 1.00 & (reference) \\
\hline & & Steady moderate & 24 & 1.06 & $(0.68,1.64)$ & 1.02 & $(0.64,1.62)$ \\
\hline & & Decreasing & 34 & 0.95 & $(0.65,1.38)$ & 0.92 & $(0.61,1.37)$ \\
\hline & & Increasing & 21 & 0.77 & $(0.49,1.22)$ & 0.71 & $(0.44,1.14)$ \\
\hline & & Steady heavy & 0 & - & & - & \\
\hline & \multicolumn{7}{|c|}{ Year before baseline $\mathrm{e}^{\mathrm{e}}$} \\
\hline & & 0 days/week & 199 & 1.00 & (reference) & 1.00 & (reference) \\
\hline & & 1 to 4 days/week & 40 & 0.98 & $(0.70,1.39)$ & 0.79 & $(0.53,1.16)$ \\
\hline & & 5 to 7 days.week & 1 & 0.33 & $(0.05,2.37)$ & 0.36 & $(0.05,2.59)$ \\
\hline & & & & \multicolumn{2}{|c|}{$P_{\text {trend }}=0.30$} & \multicolumn{2}{|c|}{$P_{\text {trend }}=0.14$} \\
\hline
\end{tabular}

Wine

Age 30-35 years and the year before baseline ${ }^{c}$

Never

Steady moderate

Decreasing

Increasing

Steady heavy

Age 18-22 years and the year before baseline ${ }^{\mathrm{d}}$

Never

Steady moderate

Decreasing

Increasing

Steady heavy

Year before baseline $\mathrm{e}^{\mathrm{e}}$

$$
\begin{aligned}
& 0 \text { days/week } \\
& 1 \text { to } 4 \text { days/week }
\end{aligned}
$$

5 to 7 days.week

Liquor
Age 30-35 years and the year before baseline ${ }^{c}$

$$
\begin{aligned}
& \text { Never } \\
& \text { Steady moderate } \\
& \text { Decreasing } \\
& \text { Increasing } \\
& \text { Steady heavy }
\end{aligned}
$$

$\begin{array}{rlccc}60 & 1.00 & (\text { reference) } & 1.00 & \begin{array}{l}\text { (reference) } \\ 65\end{array} \\ 34 & 1.15 & (0.80,1.64) & 1.19 & (0.80,1.77) \\ 53 & 1.25 & (0.82,1.92) & 1.32 & (0.82,2.11) \\ 19 & 1.25 & (0.86,1.82) & 1.29 & (0.86,1.93) \\ & 1.54 & (0.91,2.62) & 1.75 & (1.00,3.04) \\ 71 & & & & \\ 29 & 1.00 & (\text { reference) } & 1.00 & \text { (reference) } \\ 20 & 0.98 & (0.63,1.52) & 1.01 & (0.62,1.63) \\ 113 & 1.48 & (0.89,2.45) & 1.73 & (1.00,2.99) \\ 5 & 1.29 & (0.95,1.74) & 1.38 & (0.99,1.92) \\ & 2.47 & (0.99,6.19) & 2.76 & (1.09,7.00) \\ 91 & & & & \\ 97 & 1.00 & (\text { reference) } & 1.00 & (\text { reference) } \\ 37 & 1.19 & (0.89,1.60) & 1.23 & (0.89,1.71) \\ & 1.34 & (0.91,1.98) & 1.45 & (0.96,2.20) \\ & P_{\text {trend }}= & 0.18 & P_{\text {trend }}=0.11\end{array}$

\begin{tabular}{rrrrr}
110 & 1.00 & (reference) & 1.00 & (reference) \\
47 & 1.13 & $(0.80,1.60)$ & 1.12 & $(0.76,1.63)$ \\
50 & 1.04 & $(0.74,1.46)$ & 0.97 & $(0.67,1.41)$ \\
21 & 1.11 & $(0.69,1.77)$ & 1.04 & $(0.63,1.70)$ \\
3 & 0.49 & $(0.15,1.54)$ & 0.46 & $(0.15,1.49)$ \\
\hline
\end{tabular}


Table 3 continued

\begin{tabular}{|c|c|c|c|c|c|c|c|}
\hline Alcohol type & Time period & Drinking pattern & Cases $(n)$ & $\mathrm{RR}^{\mathrm{a}}$ & $95 \% \mathrm{CI}^{\mathrm{a}}$ & $\mathrm{RR}^{\mathrm{b}}$ & $95 \% \mathrm{CI}^{\mathrm{b}}$ \\
\hline & \multicolumn{7}{|c|}{ Age $18-22$ years and the year before baseline ${ }^{\mathrm{d}}$} \\
\hline & & Never & 126 & 1.00 & (reference) & 1.00 & (reference) \\
\hline & & Steady moderate & 32 & 1.12 & $(0.76,1.66)$ & 1.10 & $(0.71,1.69)$ \\
\hline & & Decreasing & 35 & 0.84 & $(0.57,1.23)$ & 0.76 & $(0.50,1.15)$ \\
\hline & & Increasing & 44 & 0.98 & $(0.69,1.38)$ & 0.92 & $(0.64,1.33)$ \\
\hline & & Steady heavy & 1 & 0.80 & $(0.11,5.77)$ & 0.69 & $(0.10,5.04)$ \\
\hline & \multicolumn{7}{|c|}{ Year before baseline $\mathrm{e}^{\mathrm{e}}$} \\
\hline & & 0 days/week & 169 & 1.00 & (reference) & 1.00 & (reference) \\
\hline & & 1 to 4 days/week & 57 & 1.13 & $(0.83,1.53)$ & 1.10 & $(0.78,1.55)$ \\
\hline & & 5 to 7 days.week & 13 & 1.00 & $(0.57,1.78)$ & 0.73 & $(0.35,1.51)$ \\
\hline & & & & \multicolumn{2}{|c|}{$P_{\text {trend }}=0.92$} & \multicolumn{2}{|c|}{$P_{\text {trend }}=0.44$} \\
\hline
\end{tabular}

$\overline{{ }^{a}}$ Adjusted for race, total energy intake, parity, oral contraceptive use, strenuous exercise, and menopausal status/hormone therapy use; stratified by age at baseline

b Additionally adjusted for drinking patterns of other alcohol types across the same time periods

c Among women over age 35 years at baseline with non-missing data on alcohol consumption at ages 30-35 years

d Among women over age 22 years at baseline with non-missing data on alcohol consumption at ages 18-22 years

e Excluding women with missing data on number of drinking days per week

race, higher total caloric intake, nulliparity, ever-use of oral contraceptives, more physical activity, ever-use of HT, higher SES or median family income, residence in the Greater San Francisco Bay Area, lower body mass index, ever-smoking of cigarettes, and higher intake of coffee and/or tea were significantly associated with drinking at least one daily glass of wine, compared to none (data not shown). However, the positive association between wine consumption and risk of ovarian cancer remained statistically significant even after adjustment for these characteristics (data not shown).

To further explore the relationship between wine consumption and ovarian cancer risk, we stratified the association by various potential effect modifiers, including demographic characteristics and ovarian cancer risk factors (Table 4). There was no statistically significant heterogeneity in the association between intake of alcohol from wine at baseline and risk of ovarian cancer by any of the factors examined. Among peri-/ post-menopausal women, we observed no association with wine intake among those who had never used HT, nor among those who used combined estrogen-progestin HT, adjusting for duration of HT use. In contrast, women who used unopposed estrogen HT were at twice the risk of ovarian cancer if they drank one glass of wine per day at baseline, compared to wine non-drinkers. The association was especially strong among women who used estrogen-only HT for over 5 years $(\mathrm{RR}=2.39$ [95\% CI 0.97-5.89], $P_{\text {trend }}=0.02$ ), whereas there was no such association among women who used combination HT for over 5 years $(\mathrm{RR}=1.34$ [95\% CI 0.51-3.54], $\left.P_{\text {trend }}=0.29\right)$. We also found that the women in the highest quartile of SES statewide were at double the risk of ovarian cancer if they drank at least a glass per day of wine, compared to none, whereas there was no such association among women in the lower three quartiles of SES.

In secondary analyses, we examined the associations between alcohol consumption and risk of ovarian cancer among only women who were peri- or postmenopausal at baseline ( $46 \%$ of the study population, $75 \%$ of cases). In this group, we observed the same lack of a significant association with total alcohol, beer, or liquor intake, along with a significant positive association with baseline wine intake. Likewise, when we restricted the case population to invasive ovarian cancer $(90 \%$ of cases) or to serous ovarian cancer ( $45 \%$ of cases), baseline intake of alcohol from wine, but not from other sources, was associated with significantly increased risk of ovarian cancer.

\section{Discussion}

The lack of association between overall alcohol consumption and risk of ovarian cancer in our study is consistent with most previous studies [3-18], including a pooled analysis of 10 prospective cohorts [19]. Alcohol consumption during ages 18-22 years, ages 30-35 years, or the year before baseline-whether from beer, liquor, or all sources combined-was unrelated to ovarian cancer development. Additionally, changes in beer, liquor, or total alcohol consumption over time, as well as frequency of drinking in the year before baseline, did not affect ovarian cancer risk in this cohort.

In contrast, average consumption of at least one glass per day of wine in the year before baseline was 
Table 4 Stratified relative risks (RRs) and 95\% confidence intervals (CIs) for associations between wine intake in the year before baseline and risk of ovarian cancer within participant subgroups

\begin{tabular}{|c|c|c|c|c|c|c|c|c|c|c|}
\hline \multirow[t]{3}{*}{ Characteristic (at baseline) } & \multicolumn{8}{|c|}{ Alcohol consumption from wine } & \multirow[t]{3}{*}{$P_{\text {trend }}$} & \multirow{3}{*}{$\begin{array}{l}P_{\text {heterogeneity }} \\
\text { between subgroups }\end{array}$} \\
\hline & \multicolumn{2}{|l|}{ None } & \multicolumn{3}{|c|}{$<11.1 \mathrm{~g} /$ day } & \multicolumn{3}{|c|}{$\geq 11.1 \mathrm{~g} /$ day } & & \\
\hline & Cases & $\mathrm{RR}^{\mathrm{a}}$ & Cases & $\mathrm{RR}^{\mathrm{a}}$ & $(95 \% \mathrm{CI})^{\mathrm{a}}$ & Cases & $\mathrm{RR}^{\mathrm{a}}$ & $(95 \% \mathrm{CI})^{\mathrm{a}}$ & & \\
\hline \multicolumn{11}{|l|}{ Age } \\
\hline$\leq$ Median (50 years) & 23 & 1.00 & 27 & 1.07 & $(0.58,1.99)$ & 12 & 1.43 & $(0.67,3.04)$ & 0.34 & \\
\hline$>$ Median & 68 & 1.00 & 72 & 1.10 & $(0.76,1.57)$ & 51 & 1.62 & $(1.09,2.39)$ & 0.01 & 0.95 \\
\hline \multicolumn{11}{|l|}{ Parity } \\
\hline Nulliparous & 18 & 1.00 & 26 & 1.34 & $(0.69,2.62)$ & 13 & 1.56 & $(0.71,3.40)$ & 0.31 & \\
\hline Parous & 71 & 1.00 & 73 & 1.05 & $(0.73,1.50)$ & 48 & 1.57 & $(1.06,2.34)$ & 0.02 & 0.61 \\
\hline \multicolumn{11}{|l|}{ Oral contraceptive use } \\
\hline Never & 45 & 1.00 & 39 & 1.00 & $(0.62,1.61)$ & 29 & 1.70 & $(1.02,2.82)$ & 0.03 & \\
\hline Ever & 22 & 1.00 & 14 & 0.76 & $(0.37,1.58)$ & 14 & 1.78 & $(0.85,3.72)$ & 0.09 & 0.54 \\
\hline \multicolumn{11}{|l|}{ Lifetime strenuous physical activity } \\
\hline$\leq$ Median (1.4 hours/week) & 61 & 1.00 & 58 & 1.07 & $(0.72,1.59)$ & 40 & 1.68 & $(1.09,2.59)$ & 0.01 & \\
\hline >Median (1.4 hours/week) & 30 & 1.00 & 41 & 1.11 & $(0.66,1.86)$ & 23 & 1.39 & $(0.77,2.50)$ & 0.26 & 0.55 \\
\hline \multicolumn{11}{|l|}{ Menopausal status } \\
\hline Pre-menopausal & 21 & 1.00 & 20 & 0.83 & $(0.42,1.65)$ & 10 & 1.24 & $(0.55,2.83)$ & 0.53 & \\
\hline Peri-/Post-menopausal & 66 & 1.00 & 72 & 1.16 & $(0.80,1.66)$ & 51 & 1.72 & $(1.16,2.55)$ & 0.01 & 0.86 \\
\hline \multicolumn{11}{|l|}{ Hormone therapy (HT) use } \\
\hline None ${ }^{\mathrm{b}}$ & 21 & 1.00 & 22 & 1.27 & $(0.64,2.51)$ & 9 & 1.20 & $(0.51,2.78)$ & 0.73 & \\
\hline $\begin{array}{l}\text { Combination } \\
\text { estrogen + progestin } \mathrm{HT}^{\mathrm{b}}\end{array}$ & 23 & 1.00 & 18 & 0.69 & $(0.35,1.37)$ & 16 & 1.17 & $(0.58,2.34)$ & 0.45 & \\
\hline Estrogen-only $\mathrm{HT}^{\mathrm{b}}$ & 16 & 1.00 & 19 & 1.27 & $(0.62,2.61)$ & 15 & 2.03 & $(0.95,4.35)$ & 0.06 & 0.37 \\
\hline \multicolumn{11}{|l|}{ Region of residence } \\
\hline Greater Bay/Southern Coastal regions & 45 & 1.00 & 62 & 1.20 & $(0.79,1.84)$ & 38 & 1.69 & $(1.06,2.71)$ & 0.02 & \\
\hline Other California regions & 46 & 1.00 & 37 & 0.94 & $(0.59,1.52)$ & 25 & 1.46 & $(0.87,2.48)$ & 0.12 & 0.41 \\
\hline \multicolumn{11}{|l|}{ Type of residence } \\
\hline Rural/town/small city & 35 & 1.00 & 34 & 1.23 & $(0.74,2.06)$ & 23 & 1.77 & $(1.01,3.11)$ & 0.05 & \\
\hline Metropolitan suburban/urban & 54 & 1.00 & 65 & 1.04 & $(0.70,1.55)$ & 40 & 1.51 & $(0.97,2.37)$ & 0.05 & 0.77 \\
\hline \multicolumn{11}{|c|}{ Statewide percentile of socioeconomic status in census block group ${ }^{c}$} \\
\hline Lower $75 \%$ & 57 & 1.00 & 50 & 1.09 & $(0.72,1.67)$ & 24 & 1.35 & $(0.81,2.27)$ & 0.25 & \\
\hline Upper $25 \%$ & 32 & 1.00 & 49 & 1.16 & $(0.72,1.88)$ & 39 & 1.96 & $(1.19,3.24)$ & 0.004 & 0.43 \\
\hline \multicolumn{11}{|l|}{ Body mass index } \\
\hline$\leq$ Median $\left(23.5 \mathrm{~kg} / \mathrm{m}^{2}\right)$ & 33 & 1.00 & 46 & 1.31 & $(0.81,2.11)$ & 32 & 1.64 & $(0.97,2.76)$ & 0.07 & \\
\hline$>$ Median & 53 & 1.00 & 52 & 0.97 & $(0.63,1.49)$ & 27 & 1.48 & $(0.89,2.45)$ & 0.10 & 0.69 \\
\hline \multicolumn{11}{|l|}{ Cigarette smoking status } \\
\hline Never & 66 & 1.00 & 61 & 1.05 & $(0.71,1.56)$ & 36 & 1.77 & $(1.13,2.78)$ & 0.01 & \\
\hline Ever & 25 & 1.00 & 38 & 1.19 & $(0.69,2.03)$ & 27 & 1.42 & $(0.80,2.50)$ & 0.24 & 0.57 \\
\hline \multicolumn{11}{|l|}{ Dietary folate intake } \\
\hline$\leq$ Median $(307.1 \mu \mathrm{g} / \text { day })^{\mathrm{d}}$ & 23 & 1.00 & 21 & 0.73 & $(0.37,1.43)$ & 13 & 1.15 & $(0.54,2.44)$ & 0.55 & \\
\hline$>$ Median $^{\text {d }}$ & 21 & 1.00 & 23 & 1.16 & $(0.60,2.24)$ & 10 & 1.17 & $(0.51,2.66)$ & 0.75 & 0.74 \\
\hline \multicolumn{11}{|l|}{ Total folate intake } \\
\hline$\leq$ Median $(473.0 \mu \mathrm{g} / \text { day })^{\mathrm{e}}$ & 43 & 1.00 & 46 & 1.07 & $(0.68,1.70)$ & 25 & 1.34 & $(0.78,2.30)$ & 0.27 & \\
\hline$>$ Median $^{\mathrm{e}}$ & 41 & 1.00 & 48 & 1.20 & $(0.77,1.89)$ & 37 & 2.07 & $(1.29,3.35)$ & 0.002 & 0.43 \\
\hline
\end{tabular}

a Adjusted for race, total energy intake, parity, oral contraceptive use, strenuous exercise, menopausal status/hormone therapy use, and consumption of beer and liquor in the past year; stratified by age at baseline

b Including peri-/post-menopausal women only

c See Methods for definition

${ }^{\mathrm{d}}$ Excluding all multivitamin users (i.e., women consuming supplemental folate)

e Excluding short-term multivitamin users (i.e., women consuming supplemental folate for $<2$ years) 
associated with elevated risk of ovarian cancer, while wine intake at ages 30-35 years or 18-22 years was associated with nonsignificantly increased risk. The positive association with baseline wine intake persisted after adjustment for total alcohol intake, suggesting that the apparent effect of wine on ovarian cancer risk was independent of alcohol content. The association was also unchanged by further adjustment for characteristics and behaviors associated with wine drinking in this cohort, and was not significantly modified by reproductive characteristics, demographic factors, or folate intake. However, there was a significant positive association between wine consumption and ovarian cancer risk among peri-/post-menopausal women who used unopposed estrogen HT, whereas there was no such association among peri-/post-menopausal women who did not use HT or used combined estrogen-progestin HT. Wine consumption was also associated with increased ovarian cancer risk among women of high SES, but not among women of relatively low SES.

To our knowledge, no other study of ovarian cancer has examined alcohol consumption at various ages or drinking patterns over time. A positive association between wine consumption and risk of ovarian cancer has previously been detected in two case-control studies [15, 49], although both of those studies reported a positive association with total alcohol intake as well. In contrast, two case-control studies $[14,24]$ and one cohort study (among women with high dietary folate intake) [17] found an inverse association between wine consumption and ovarian cancer risk. However, there was no association with wine (or total alcohol) consumption in other studies [13, 18, 19].

Few previous studies have examined modification of the association between alcohol consumption and risk of ovarian cancer. A pooled analysis of 529,638 women, including 2,001 incident ovarian cancer cases, found no interaction between alcohol intake and oral contraceptive or HT use, parity, menopausal status, folate intake, BMI, or smoking [19]. In contrast, a case-control study found that an inverse association between wine consumption and ovarian cancer risk was significantly stronger in women who were more highly educated, had never smoked, or had used oral contraceptives-characteristics that describe the majority of CTS cohort members-but found no heterogeneity by menopausal status, BMI, or HT use [24]. Unlike us, others reported an interaction with folate intake $[16,17,25]$ or heterogeneity by histologic subtype of ovarian cancer $[14,15]$, although others did not $[3,12,19,24]$. We lacked sufficient cases to perform detailed analyses of ovarian cancer histologic subtypes other than the most common serous type.
The restriction of the positive association between wine intake and ovarian cancer risk to peri-/post-menopausal women taking estrogen-only HT suggests a biological mechanism involving estrogen. Likewise, the restriction of the association to women of high SES may reflect reproductive characteristics and/or HT use favoring increased estrogen levels in higher-SES women. A high background level of circulating estrogen, compounded by an upsurge of estrogen resulting from alcohol consumption [1], may promote ovarian carcinogenesis. Furthermore, the lack of an interaction between wine intake and use of estrogen-progestin HT could be explained by a protective effect of progestin against ovarian cancer. Phytochemicals, such as resveratrol, in red wine have been considered as promising cancer preventive agents due to their anti-estrogenic, antioxidant, anti-proliferative, and other anti-carcinogenic effects [50, 51]. However, such phytochemicals have multifarious effects, including pro-estrogenic activity and possible genotoxicity [52-54]; thus, both the potentially beneficial and potentially harmful effects of phytochemicals on cancer development must be considered together. In the present analysis, we were not able to distinguish red from white wine intake.

Our findings should be interpreted in light of some limitations. It is possible that the observed positive association between wine consumption and ovarian cancer risk was due to confounding by unmeasured characteristics beyond those for which we attempted to adjust, or by residual confounding or chance. Our efforts to fully evaluate effect modifiers or confounders of the association between wine consumption and ovarian cancer risk were partly hampered by the lack of heterogeneity in some characteristics among CTS participants. The proportion of women who drank beer or liquor was low, although the range of total alcohol intake was adequate to reveal a significant positive association with risk of breast cancer in an earlier analysis [37]. Even though reporting of alcohol intake in the past year using our questionnaire has been validated, we were not able to assess the reliability or validity of self-reported alcohol consumption at earlier ages, which may be difficult to recall, especially among older women. Misclassification of distant past alcohol intake may explain the absence of a significant association between wine drinking at earlier ages and ovarian cancer risk. Nevertheless, it is unlikely that any exposure misclassification differed systematically between ovarian cancer cases and non-cases, since all information was assessed prospectively.

In summary, given the lack of association between overall alcohol consumption and ovarian cancer risk in our study, further investigations are necessary to 
determine whether ingredients of wine, but not beer or liquor, foster ovarian cancer development; whether correlates of wine drinking not measured or imperfectly measured in our study population are associated with ovarian cancer risk; or if an association is largely due to the apparent interaction between wine consumption and unopposed estrogen HT use and/or other characteristics of women of high SES. If the observed association between wine drinking and ovarian cancer risk is due to confounding, then there may exist an as-yet unidentified ovarian cancer risk factor that is also associated with wine drinking. On the other hand, if the interactions with estrogen HT use and SES are confirmed, there may be a biological basis for the increased risk of ovarian cancer among wine drinkers with high endogenous and exogenous estrogen levels. However, if alcohol consumption is indeed unrelated to ovarian cancer development but positively associated with the risk of breast cancer, then differences in the hormonal and non-hormonal triggers between these two malignancies may help us understand the carcinogenic effects of alcohol on hormonally responsive tissue. Further understanding of the complex relationships among steroid hormone levels, metabolism of alcohol and wine, and carcinogenesis will help clarify what role, if any, alcohol and wine play in the development of ovarian cancer.

Acknowledgment This research was supported by grants R03 CA113024 and R01 CA77398 from the National Cancer Institute, and contract 97-10500 from the California Breast Cancer Research fund. The funding sources did not contribute to the design or conduct of the study, nor to the writing or submission of this manuscript. The collection of cancer incidence data used in this study was supported by the California Department of Health Services as part of the statewide cancer reporting program mandated by California Health and Safety Code Section 103885; the National Cancer Institute's Surveillance, Epidemiology and End Results Program under contract N01-PC-35136 awarded to the Northern California Cancer Center, contract N01-PC-35139 awarded to the University of Southern California, and contract N02-PC-15105 awarded to the Public Health Institute; and the Centers for Disease Control and Prevention's National Program of Cancer Registries, under agreement \#U55/ CCR921930-02 awarded to the Public Health Institute. The ideas and opinions expressed herein are those of the author(s) and endorsement by the State of California, Department of Health Services, the National Cancer Institute, and the Centers for Disease Control and Prevention or their contractors and subcontractors is not intended nor should be inferred.

\section{References}

1. Singletary KW, Gapstur SM (2001) Alcohol and breast cancer: review of epidemiologic and experimental evidence and potential mechanisms. JAMA 286(17):2143-51
2. Brekelmans CT (2003) Risk factors and risk reduction of breast and ovarian cancer. Curr Opin Obstet Gynecol 15(1):63-8

3. Riman T, Dickman PW, Nilsson S, Nordlinder H, Magnusson CM, Persson IR (2004) Some life-style factors and the risk of invasive epithelial ovarian cancer in Swedish women. Eur J Epidemiol 19(11):1011-9

4. Gwinn M, Webster L, Lee N, Layde P, Rubin G (1986) Alcohol consumption and ovarian cancer risk. Am J Epidemiol 123(5):759-66

5. Whittemore A, Wu M, Pafrfenbarger RJ, et al (1988) Personal and environmental chararcteristics related to epithelial ovarian cancer. II. Exposures to talcum powder, tobacco, alcohol, and coffee. Am J Epidemiol 128(6):122840

6. Hartge P, Schiffman MH, Hoover R, McGowan L, Lesher L, Norris HJ (1989) A case-control study of epithelial ovarian cancer. Am J Obstet Gynecol 161(1):10-6

7. Adami HO, McLaughlin JK, Hsing AW, et al (1992) Alcoholism and cancer risk: a population-based cohort study. Cancer Causes Control 3(5):419-25

8. Polychronopoulou A, Tzonou A, Hsieh CC, et al (1993) Reproductive variables, tobacco, ethanol, coffee and somatometry as risk factors for ovarian cancer. Int $\mathrm{J}$ Cancer 55(3):402-7

9. Tonnesen H, Moller H, Andersen JR, Jensen E, Juel K (1994) Cancer morbidity in alcohol abusers. Br J Cancer 69(2):327-32

10. Nandakumar A, Anantha N, Dhar M, et al (1995) A casecontrol investigation on cancer of the ovary in Bangalore, India. Int J Cancer 63(3):361-5

11. Sigvardsson S, Hardell L, Przybeck TR, Cloninger R (1996) Increased cancer risk among Swedish female alcoholics. Epidemiology 7(2):140-3

12. Kuper H, Titus-Ernstoff L, Harlow BL, Cramer DW (2000) Population based study of coffee, alcohol and tobacco use and risk of ovarian cancer. Int J Cancer 88(2):313-8

13. Tavani A, Gallus S, Dal Maso L, et al (2001) Coffee and alcohol intake and risk of ovarian cancer: an Italian casecontrol study. Nutr Cancer 39(1):29-34

14. Goodman MT, Tung KH (2003) Alcohol consumption and the risk of borderline and invasive ovarian cancer. Obstet Gynecol 101(6):1221-8

15. Modugno F, Ness RB, Allen GO (2003) Alcohol consumption and the risk of mucinous and nonmucinous epithelial ovarian cancer. Obstet Gynecol 102(6):1336-43

16. Larsson SC, Giovannucci E, Wolk A (2004) Dietary folate intake and incidence of ovarian cancer: the Swedish Mammography Cohort. J Natl Cancer Inst 96(5):396402

17. Larsson SC, Wolk A (2004) Wine consumption and epithelial ovarian cancer. Cancer Epidemiol Biomarkers Prev 13(11Pt1):1823; author reply -4

18. Schouten LJ, Zeegers MP, Goldbohm RA, van den Brandt PA (2004) Alcohol and ovarian cancer risk: results from the Netherlands Cohort Study. Cancer Causes Control 15(2):201-9

19. Genkinger JM, Hunter DJ, Spiegelman D, et al (2006) Alcohol intake and ovarian cancer risk: a pooled analysis of 10 cohort studies. Br J Cancer 94(5):757-62

20. Tzonou A, Day NE, Trichopoulos D, et al (1984) The epidemiology of ovarian cancer in Greece: a case-control study. Eur J Cancer Clin Oncol 20(8):1045-52

21. La Vecchia C (1989) Nutritional factors and cancers of the breast, endometrium and ovary. Eur J Cancer Clin Oncol 25(12):1945-51 
22. Kato I, Tominaga S, Terao C (1989) Alcohol consumption and cancers of hormone-related organs in females. Jpn J Clin Oncol 19(3):202-7

23. Lagiou $\mathrm{P}, \mathrm{Ye} \mathrm{W}, \mathrm{Wedren} \mathrm{S}$, et al (2001) Incidence of ovarian cancer among alcoholic women: a cohort study in Sweden. Int J Cancer 91(2):264-6

24. Webb PM, Purdie DM, Bain CJ, Green AC (2004) Alcohol, wine, and risk of epithelial ovarian cancer. Cancer Epidemiol Biomarkers Prev 13(4):592-9

25. Kelemen LE, Sellers TA, Vierkant RA, Harnack L, Cerhan JR (2004) Association of folate and alcohol with risk of ovarian cancer in a prospective study of postmenopausal women. Cancer Causes Control 15(10):1085-93

26. Ho SM (2003) Estrogen, progesterone and epithelial ovarian cancer. Reprod Biol Endocrinol 1:73

27. Reichman ME, Judd JT, Longcope C, et al (1993) Effects of alcohol consumption on plasma and urinary hormone concentrations in premenopausal women. J Nat Canc Inst 85(9):722-3

28. Sarkola T, Fukunaga T, Makisalo H, Peter Eriksson CJ (2000) Acute effect of alcohol on androgens in premenopausal women. Alcohol Alcohol 35(1):84-90

29. Dorgan JF, Reichman ME, Judd JT, et al (1994) The relation of reported alcohol ingestion to plasma levels of estrogens and androgens in premenopausal women (Maryland, United States). Cancer Causes Control 5(1):53-60

30. Grodstein F, Goldman MB, Cramer DW (1994) Infertility in women and moderate alcohol use. Am J Public Health 84(9):1429-32

31. Jensen TK, Hjollund NH, Henriksen TB, et al (1998) Does moderate alcohol consumption affect fertility? Follow-up study among couples planning first pregnancy. BMJ 317(7157):505-10

32. Cooper GS, Sandler DP, Whelan EA, Smith KR (1996) Association of physical and behavioral characteristics with menstrual cycle patterns in women age 29-31 years. Epidemiology 7(6):624-8

33. Ginsburg ES, Walsh BW, Gao X, Gleason RE, Feltmate C, Barbieri RL (1995) The effect of acute ethanol ingestion on estrogen levels in postmenopausal women using transdermal estradiol. J Soc Gynecol Investig 2(1):26-9

34. Ginsburg ES, Mello NK, Mendelson JH, et al (1996) Effects of alcohol ingestion on estrogens in postmenopausal women. JAMA 276(21):1747-51

35. Purohit V (1998) Moderate alcohol consumption and estrogen levels in postmenopausal women: a review. Alcohol Clin Exp Res 22(5):994-7

36. Horn-Ross PL, Hoggatt KJ, West DW, et al (2002) Recent diet and breast cancer risk: the California Teachers Study (USA). Cancer Causes Control 13(5):407-15

37. Horn-Ross PL, Canchola AJ, West DW, et al (2004) Patterns of alcohol consumption and breast cancer risk in the California Teachers Study cohort. Cancer Epidemiol Biomarkers Prev 13(3):405-11
38. Bernstein L, Allen M, Anton-Culver H, et al (2002) High breast cancer incidence rates among California teachers: results from the California Teachers Study (United States). Cancer Causes Control 13(7):625-35

39. Block G, Hartman AM, Dresser CM, Carroll MD, Gannon J, Gardner L (1986) A data-based approach to diet questionnaire design and testing. Am J Epidemiol 124(3):453-69

40. Block G, Subar AF (1992) Estimates of nutrient intake from a food frequency questionnaire: the 1987 National Health Interview Survey. J Am Diet Assoc 92(8):969-77

41. Block G, Thompson FE, Hartman AM, Larkin FA, Guire KE (1992) Comparison of two dietary questionnaires validated against multiple dietary records collected during a 1year period. J Am Diet Assoc 92(6):686-93

42. Block G, Woods M, Potosky A, Clifford C (1990) Validation of a self-administered diet history questionnaire using multiple diet records. J Clin Epidemiol 43(12):1327-35

43. Chen VW, Ruiz B, Killeen JL, Cote TR, Wu XC, Correa CN (2003) Pathology and classification of ovarian tumors. Cancer 97(10Suppl):2631-42

44. Reynolds P, Hurley S, Goldberg DE, et al (2004) Regional variations in breast cancer among California teachers. Epidemiology 15(6):746-54

45. Reynolds P, Hurley SE, Quach AT, et al (2005) Regional variations in breast cancer incidence among California women, 1988-1997. Cancer Causes Control 16(2):139-50

46. Kwong SL, Perkins CI, Morris CR, Cohen R, Allen M, Wright WE (2001) Cancer in California: 1988-1999. Sacramento, CA: California Department of Health Services, Cancer Surveillance Section

47. Hosmer DW Jr, Lemeshow S (1999) Applied survival analysis. Regression modeling of time to event data. John Wiley \& Sons, Inc. New York

48. Breslow NE, Day NE (1987) Statistical methods in cancer research. Volume 2 - The design and analysis of cohort studies. International Agency for Research on Cancer Lyon

49. La Vecchia C, Negri E, Franceschi S, Parazzini F, Gentile A, Fasoli M (1992) Alcohol and epithelial ovarian cancer. J Clin Epidemiol 45(9):1025-30

50. Jang M, Cai L, Udeani GO, et al (1997) Cancer chemopreventive activity of resveratrol, a natural product derived from grapes. Science 275(5297):218-20

51. Cornwell T, Cohick W, Raskin I (2004) Dietary phytoestrogens and health. Phytochemistry 65(8):995-1016

52. Wietrzyk J, Grynkiewicz G, Opolski A (2005) Phytoestrogens in cancer prevention and therapy-mechanisms of their biological activity. Anticancer Res 25(3c):2357-66

53. Stopper H, Schmitt E, Kobras K (2005) Genotoxicity of phytoestrogens. Mutat Res 574(1-2):139-55

54. Schmitt E, Lehmann L, Metzler M, Stopper H (2002) Hormonal and genotoxic activity of resveratrol. Toxicol Lett 136(2):133-42 